\title{
THE CHALLENGES OF APPLYING GROUNDED THEORY IN INNOVATION AND PRODUCT DEVELOPMENT: A CASE STUDY IN AGILE PROJECT MANAGEMENT
}

Carolina Rodrigues Reigado (carolinareigado@gmail.com) - Production Engineering Department, University of Sao Paulo

Marcelle La Guardia Lara de Castro (marcelle.laguardia.lara@gmail.com) - Production Engineering Department, Federal University of Minas Gerais

Ana Valéria Carneiro Dias (anaval@ufmg.br) - Production Engineering Department, Federal University of Minas Gerais

Daniel Capaldo Amaral (amaral@sc.usp.br) - Production Engineering Department, University of Sao Paulo

\begin{abstract}
Grounded Theory (GT) is a qualitative method applied especially in studies of human and social aspects. The application of GT is challenging, since the method does not utilize previously developed hypothesis and suggests the adoption of a wide scope of research. More than that, the literature of GT applied in Innovation and Product Development (PD) is still in early stages, with little evidence on details of how applying the method. This paper presents the challenges regarding GT usage faced by a research on improvisation in Agile Project Management (APM) teams. A brief literature review was applied in order to identify GT protocols from different areas, a protocol was developed for the improvisation's research and the difficulties faced by the researchers were written down in a case study. The definition of the phenomenon, for instance, is one of the critical barriers identified by the authors. The examples found in literature, from human and social aspects, were not sufficient to guide author regarding this aspect. The strategy adopted was to create a practical definition of the phenomenon, including a trigger and an ending state. Other problems, such as the consolidation of the data analysis, are also discussed. Finally, it is expected that the analysis will be useful for researchers interested in adopting GT for PD and innovation or similar areas.
\end{abstract}

Keywords: Grounded Theory, Innovation, Product Development, Project Management, protocol.

Area: Management of product and service development projects

\section{INTRODUCTION}

Grounded Theory (GT) is a qualitative research method which aims to create theory based on systematically collected and analysed data (Glaser and Strauss, 1967). Therefore, researches following GT are not based on predefined hypothesis, but grounded to the data. GT includes some techniques for systematically analysing the data and achieving the creation of theory, 
such as coding and constant comparison. By doing so, it is possible to create theories that are closely related to the context in which data were collected (GLASER and STRAUSS, 1967).

GT's methods are a set of principles, practices and flexible guidelines to support data analysis, considering that the indication of the research's next steps will emerge from the data (CHARMAZ, 2006). Additionally, Glaser e Strauss (1967) list essential elements of GT. Thus, there is not a rigid set of rules and methodological requirements, but a non-linear process that is developed along the research itself.

Although the authors highlight the importance of GT's flexibility (CHARMAZ, 2006; GLASER and STRAUSS, 1967), the main challenge of researchers intending to apply GT is how to manage it. Unreasonable flexibility could result in unproductive or poor methodological procedures at many points of the research, such as the transition between the coding stages and the delimitation of the phenomenon to be studied.

The product development (PD) process is "the set of activities beginning with the perception of a market opportunity and ending in the production, sale, and delivery of a product" (ULRICH and EPPINGER, 2012, p. 2). Rozenfeld et al (2006) mentions that the PD process is also guided by the company's strategy and technological restriction, and also embraces activities after the sale, such as managing the required changes in the product and planning the product's end of life.

In this context, innovative projects generally involve unknown requirements and risks, which leads to the necessity of the team having the ability to adapt the plan according to changes. (CONFORTO, REBENTISCH and AMARAL, 2016). In fact, Conforto, Rebentisch and Amaral (2016) conducted a survey that confirms this idea, indicating that projects with fluid requirements are more likely to present higher levels of improvisation.

This paper investigates the application of GT at the PD and innovation context, through the analysis of a specific research case, a case study on improvisation in agile teams. The study involves a software business-to-business company and it is still in course.

The paper is organized into five sections. Firstly, in section two, GT will be presented, including the steps and techniques together with a brief historical view. Secondly, examples from literature of application of GT in Product Development (PD) will be presented. Section four comprises the method of this study. The challenges and strategies applied in the agile teams research will be presented in section five. Finally, in section six, final considerations, summarizes the contributions of the study and list gaps for future researches to fulfil.

\section{GROUNDED THEORY}

At a time when quantitative methods were almost exclusives in Science, Barley Glaser e Anselm Strauss applied scientific rigour on qualitative research, based on criteria such as the significance of data and not only the extension or reliability (Tarozzi, 2011). By defending the development of theory connected to the concrete ground of data instead of deducting hypothesis based on existent theories, the authors caused discussion in the context of social sciences (CHARMAZ, 2006).

Glaser e Strauss (1967) conceptualized GT as a general and comparative analysis method, while Charmaz (2006) referred to it as a constellation of methods. According to Tarozzi (2011) it consists of the theoretical look over data collection and analysis, and the concrete instruments for the stages, aiming to theorizing based on the data grounding and making conceptual regularities emerge during the analysis. GT, therefore, is a tentative of dealing with inexact issues (TAROZZI, 2011). 
Even though GT does not present an unequivocal theoretical framework, it is ontologically linked to Pragmatism and Symbolic Interactionism, as it considers social reality as dynamic and social acting as a product of continuous, symbolic and intentional changes among actors (TAROZZI, 2011; CORBIN AND STRAUSS, 1990). Therefore, GT is utilized for supporting the build and the coding of data, since its main contribution is connected to the analytical practices such as coding, constant comparison and memos.

Tarozzi (2011) describes the process of GT as the following phases: identification of an investigation area, definition of the research question, definition of methods and tools, initial coding, theoretical sampling, focused coding, writing of memos, theoretical coding, writing of the report and evaluation of the research.

Coding is the set of procedures and techniques for conceptualizing the data (Tarozzi, 2011). It creates the bones of the analysis, being the fundamental link between data collection and the development of the emerging theory (Charmaz, 2006). Moreover, Glaser and Strauss (1967) highlight the importance of using emerging categories, instead of categories previously defined in literature.

When it comes to coding, authors organize the analysis in different phases. Tarozzi (2011) suggests initial coding, focused coding and theoretical coding. On the other hand, Charmaz (2006) lists four phases, adding the axial coding phase, while Corbin and Strauss (1990) suggest three: open, axial and selective. Although the authors utilize different names and number of stages, there is a strong relation between all these propositions. Even though the labels are different, the content is similar and refers to a gradual analysis in which the level of abstraction rises as coding advances. This study is based majorly on the procedure of Tarozzi (2011).

Another method, also fundamental in GT, is constant comparison. It consists of comparing data all the time, from the beginning of the analysis process until its end. Data should be compared not only when in the same transcript, but also between different transcripts and even different data collection cycles. The main objective of this comparison is to identify possible similarities and differences among data, which may contribute to either confirming or changing concepts and categories being theorized (Glaser and Strauss, 1967).

Regarding data collection it is relevant to note that, in GT, sampling is not planned previously, as in statistical based researches, and it is not isolated from analysis. Sampling is defined during the investigation, aiming to fulfil the gaps identified along the research (FLICK, 2009). By doing so, it is the data analysis that guides the definition of the sample, and not the researcher's previous conception, in a process called theoretical sampling (Glaser and Strauss, 1967).

Memos are the fundamental link for connecting the stages, determining the next steps and the theoretical sampling. Although they are partial and transient, memos constitute a valuable source for the writing of the research's results, as new ideas and insights may emerge from the process of writing memos (CHARMAZ, 2006).

When it comes to researches in Agile Project Management (APM), a study regarding selfmanagement in agile teams by Hoda, Noble and Marshall (2012) states that the use of GT is coherent and beneficial for APM researches. Because it is a qualitative methodology, these authors believe GT is especially interesting for studies focusing on human and social aspects. The same authors also point out the growing tendency use of GT in agile teams' researches. 


\section{GT ON INNOVATION AND PD FIELD}

The applications of GT found in the early books and articles relate to topics such as the experience of dying in a hospital (Glaser and Strauss, 1967), people suffering from diseases (Charmaz, 2006) and the experience of watching television on families with children (Tarozzi, 2011). These phenomena involve individuals in their personal lives, their perception and experience. The context of PD and Innovation, otherwise, could not be more different. It involves complex collective processes, embraced in an organizational context, with rules, procedures and use of intellectual intensive jobs, methods and techniques.

PD is deeply connected to the use of teams. Since the firstly studies conducted by Clark and Wheelright (1993) the importance of teams was pointed as crucial for understanding this process. The structure, leadership and integration among different areas' teams are some of the aspects reinforced by these authors and recent surveys as those conducted by Griffin (1997) in the United States and Barczak, Griffin and Kahn (2006), in 2003, worldwide. They have confirmed the presence of a formal process for new product development (NPD), guided by a strategy but mainly conducted by groups of people immersed in a complex relationship.

As the PD and Innovation phenomena are strongly related to teams, and not isolated individuals, the perception and feelings of a single person will not suit the PD context and the GT application must consider this aspect. That is one of the reasons that make the definition and delimitation of the research problem challenging.

Hoda, Noble and Marshall (2012) focus on the context of APM and present the steps followed during the application of GT, as exhibited in Figure 1. The steps are: choose a general research area, conduct a minor literature review, data collection (via theoretical sampling) and data analysis (iteratively): open coding, constant comparison, emergence of a core category, selective coding, memoing, sorting, theoretical coding, and write-up. The paper also mentions some challenges the authors faced, such as thinking theoretically.

Figure 1- GT steps. Source: Hoda, Noble and Marshall, 2012.

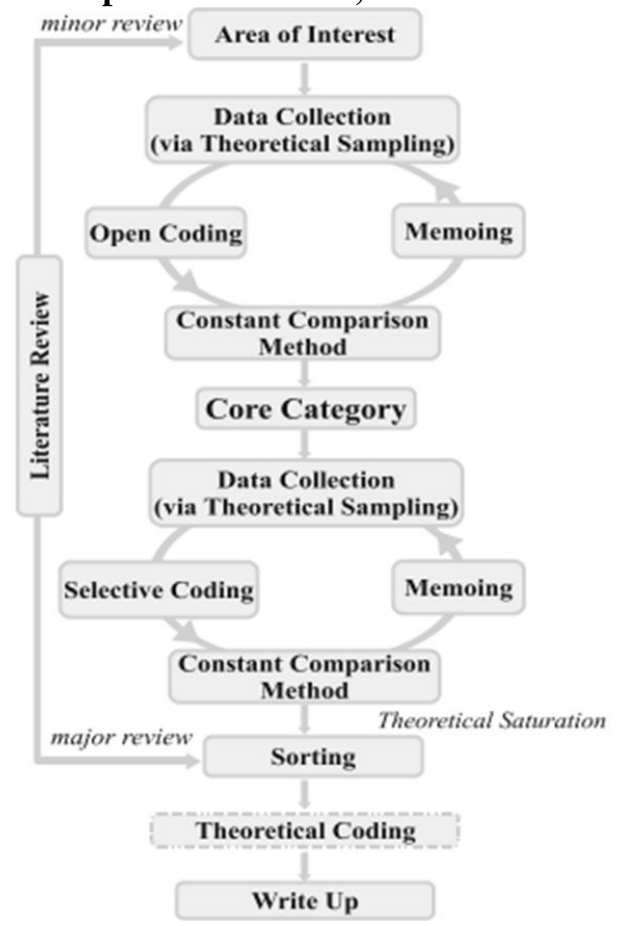


Additionally, the work of Stray, Sjøberg and Dybå (2016) also consisted of applying GT in agile teams and had daily meetings as a core category. In this case, there was a strict limit for the observation, the meeting interval. However, other aspects are not covered, as the authors did not mention the singularity of teams' perspectives and their influence in GT application, even though the study directly focused on the teams.

This paper discusses some challenges, from the experience of previous works, in a GT application at a research about improvisation in APM teams. The phenomenon of improvisation was introduced to the PD literature with the work of Moorman and Miner (1998) and, according to these authors, improvisation consists of the convergence of planning and execution of an action. Although this construct is vague on theory, it is believed to enlighten the comprehension of agile teams. The use of GT could contribute for this investigation of describing how agile teams adapt the plan during the project. The research is still in course.

\section{METHOD}

The improvisation case study involves a business-to-business (B2B) information technology (IT) enterprise that works with creation, maintenance and modification of software for other companies. Its main site is located in the nearby area of São Paulo, in Brazil, where 160 employees are stationed, half of which are software developers. The software developers work in project teams and part of the teams apply agile practices. Two teams were involved in the research and they use practices and tools originated from SCRUM, XP and Kanban methodologies, such as daily meetings, pair programming and the Kanban board.

The main objective of this case study is to improve knowledge on how agile teams improvise. The steps followed are exhibited in Figure 2. The first step to design this study was the search for GT protocols. This was not a systematic literature review, but a brief one. Protocols from different areas, such as health and APM, were found and considered. Then, based on the reading of these protocols and the GT literature, a protocol was elaborated for the APM teams' research.

Figure 2 - Steps followed by this study. Source: Created by the authors.

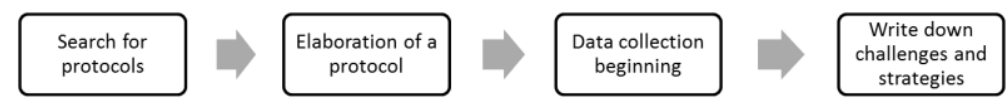

Next, the data collection was initiated. It began in November 2016 and is still in course. It has been done through observations and semi-structured interviews with project teams working with APM practices. Observations have been the major source of data, as the researcher spends hours with the teams, during meetings, pair programming sessions and routinary work, as a non-participant observer.

Although observations have been the primary source of data, individual and group interviews are also being applied. The aim is to use interviews as a complementary source of data, in order to confirm impressions caused by observations, clarify specific aspects or capture an individual's point of view about a situation. A questionnaire was developed to guide the interviews and it has been adapted depending on the case and the phase of the research. Besides, the use of group interviews has been an efficient way of collecting the teams' perception and not only individual ones. It was only possible, however, due to the proximity and favourable climate between team members. 
This research is currently in the focused coding phase. By the end of initial coding there were more than 20 transcriptions and more than 100 codes. More than that, seven categories have been developed.

Since the elaboration of the protocol until the present moment of the study the researchers faced different challenges. These challenges have been discussed and registered, as have been the strategies applied to deal with each of them. This content will be presented in next section.

\section{TWO CHALLENGES OF GT APPLICATION ON INNOVATION AND PD CONTEXT}

\subsection{Defining and delimiting the phenomenon using triggers}

The research on improvisation in agile teams is still in course and the researchers believe it could contribute to improving understanding on how agile teams adapt the project plan according to unexpected changes faced during the project. However, the challenges started to appear already in the first step of GT: definition of the area of interest. If the method is being applied in order to enhance comprehension about a phenomenon, how to define it a priori and establish the initial research's boundaries?

Glaser and Strauss (1967) focused on the experience of patients dying in hospitals. The actor, here, is one person. The study involved interviews with nurses, for instance, but the focus of the phenomenon was in an individual's experience. In this case, the definition the scope of the phenomenon may be not as challenging, since it is delimited to the individual's own perspective and perception. This could be the reason why these authors did not discuss in details how to establish the phenomenon to be studied.

When it comes to improvisation, however, the phenomenon involves the complex relationship among individuals that interact and establish plans and actions. This takes form through a set of events. This dynamic also affects the perception of each individual during time. Thus, the set of data collected and analysed is possibly wider, due to the necessity of describing the dynamics of the interaction between the actors as well as the evolution of their perception regarding the phenomenon over time.

The result was the collection of a great amount of data in the beginning of the research, including actors' perceptions and occurrences of events. How to select the right data when a part of it seemed irrelevant? This reflection brought the perception of the necessity of establishing a scope for the phenomenon, allowing for focusing the analysis and, as a consequence, generating a useful and relevant theory.

Therefore, it was necessary to establish an improvisation definition specifically for the case study instead of using definitions proposed in the literature, with the purpose of creating a scope for the analysis. According to the definition, improvisation episodes are moments when the team needs to act differently from the project's plan, or moments when the team or a team's member finds out that the plan is not coherent, and identify the need for a new solution. The phenomenon comes to an end when the team, after looking for and selecting a new solution, establishes a new plan or agreement that fits the situation, execute the action and verify its effectiveness. A trigger and a point of ending were included: the trigger is the perception of the team or a team's member of the necessity of changing the plan, while the ending point is the verification of the effectiveness of the new solution, independently of its success or unsuccess. 
Finally, by clearly marking the beginning and the ending of the phenomenon, it was possible to identify, during observations of the project team's work, the occurrences of improvisation episodes. Even though the work of Stray, Sjøberg and Dybå (2016) did not explicitly used this idea or mentioned it as a step of the methodological approach, it was implicitly part of their method, since there is a clear scope for the observations in their study, the daily meetings. For this reason, it was considered as an inspiration for the approach presented here.

In addition, a second advantage of this approach for defining the phenomenon relates to keeping track of the occurrence of the phenomenon. The researchers took advantage of the coding procedure for doing so. Specific codes were created for identifying, among the transcriptions content, when the phenomenon was occurring. By doing so, it was easy to keep track of the phenomenon occurrences and to execute constant comparison, analysing how the team acted in the different occurrence of the phenomenon.

These codes were grouped and kept isolated from the other codes in order not to interfere in the analysis. The software utilized in the research allowed for organizing the codes in groups, facilitating this approach.

\subsection{Categorizing the data}

The literature of GT determines the levels followed by the analysis. The data is organized in concepts that are, then, organized in categories, which, together with its properties and relations, compose the final theory (Glaser and Strauss, 1967). The original studies, nevertheless, do not detail the process of categorization and analysis of the categories.

Charmaz (2006) mentions that categories start to arise during focusing coding, together with the most evident themes. The author mentions a process for this: selecting the codes that have emerged in analysis as the most relevant ones, during initial coding, then comparing them to other codes and to other segments of text. Additionally, she mentions that it may be necessary to go back to open coding, iteratively. It is still not clear, however, how the researcher will proceed in the analysis.

The strategy applied in the agile teams' improvisation research was to group the concepts according to similarity and interface. For doing so, a visual map was developed, in which concepts relating to the same topic were connected to a main theme, as Figure 3 exemplifies. The main themes, created during the process of elaborating the map, were the categories. More than a result of the analysis, this map was key for the process of creating the categories, since it leads the researcher to explore possible relations among the concepts in a simple and visual manner.

Figure 3 - Visual map applied in the analysis. Source: Created by the authors.

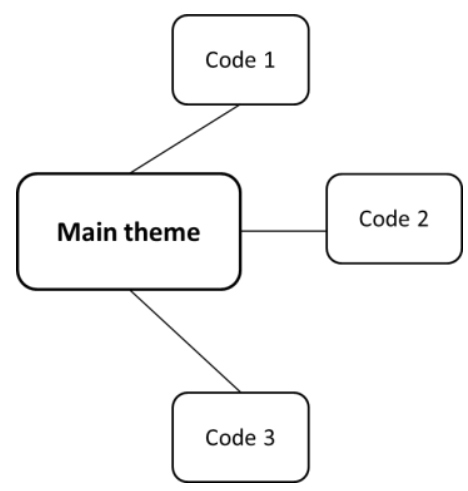


After creating the categories, a template was developed in order to describe them. This template includes the following sections: synthesis of the content, identification of characteristics and properties, hypothesis and aspects to be further investigated. The synthesis of the content includes one or two phrases summarizing the main idea of the category. The characteristics and properties include aspects that have been observed during the field study, related to the category. For every property, a verbalization obtained in the field study is mentioned, as a manner of justifying the affirmations and to make explicit the idea of the analysis being grounded to the raw data.

The third section, hypothesis, includes aspect that may have been observed in one case and that the researchers wonder if it is true for every case, or aspects that have not been observed but the researchers have some impression about. From the hypothesis, questions are elaborated, aiming to investigate deeply these assumptions. These questions are grouped in the fourth section: aspects to be further investigated.

The outputs from this process were the list of categories, the templates filled for each category and a questionnaire gathering questions originated in the analysis of each category, for being applied in the next data collection iteration.

\section{FINAL CONSIDERATIONS}

Although authors argue that GT method is in fact flexible and should be adapted to each research (Charmaz, 2006; Glaser and Strauss, 1967), the experience of applying GT in the context of PD and innovation is challenging, especially due to the collective aspect of NPD processes and the extensive use of tools and standardized procedures. The strategies presented in this study point some directions for researchers interested in applying the method, without compromising the flexibility or the grounded aspects of GT.

The strategy of delimiting the phenomenon at the beginning of the research, using a general definition with triggers and endpoints, is a new step to be considered by GT applicants on PD and innovation. It is not described in the original texts of GT but it has contributed positively for the research of improvisation in agile teams, facilitating the data analysis.

More than that, the practice of using codes for keeping track of the occurrence of the phenomenon is an interesting suggestion for future GT applications, as it allows for analysing the connection between each occurrence of the phenomenon and the other codes originated from the traditional coding process mentioned in GT literature.

Finally, the template suggested for characterizing the analytical categories is also a contribution of this study, as it allows for the researcher to consolidate initial information and visualize the gaps of information regarding the categories, contributing for the planning of the next data collection.

As highlighted earlier, the APM case study is still in course, which may imply in new challenges being faced, as focused coding is in course and theoretical coding has not been initiated yet. The further development of the categories and the description of their properties and connections between different categories have not been part of the scope of this study and may be discussed in a further one.

More than that, many questions and details regarding the application of GT in PD context are still to be investigated, such as the impact of the team members' different perspectives on the research. Future studies could investigate this aspect, as much as pointing other challenges and strategies for applying GT, enriching the discussion proposed in this paper and stimulating researchers in the use of the method. 


\section{REFERENCES}

BARCZAK, G.; GRIFFIN, A.; KAHN, K. B. PERSPECTIVE: Trends and Drivers of Success in NPD Practices: Results of the 2003 PDMA Best Practices Study. Journal of product innovation management, v. 26, p.3-33, 2006.

CHARMAZ, K. Constructing Grounded Theory: A Practical Guide Through Qualitative Analysis. London: Sage Publications, 2006.

CLARK, K. B.; WHEELRIGHT, S. C. Managing New Product and Process Development: Text and Cases. Nova York: Free Press, 1993.

CONFORTO, E. C.; REBENTISCH, E.; AMARAL, D. C. Learning the Art of Business

Improvisation. MIT Sloan, v. 57, p. 7-10, 2016.

CORBIN, J.; STRAUSS, A. Grounded Theory Research: Procedures, Canons and Evaluative Criteria. Qualitative Sociology, v. 13, n. 1, p.3-21, 1990.

FLICK, U. Uma introdução à pesquisa qualitative. 3.ed. Porto Alegre: Bookman, 2009.

GLASER, B. G.; STRAUSS, A. L. The Discovery of Grounded Theory: Strategies for Qualitative Research. Piscataway: Transaction Publishers, 1967.

GRIFFIN, Abbie. PDMA research on new product development practices: Updating trends and benchmarking best practices. Journal of product innovation management, v. 14, n. 6 , p.429-458, 1997.

HODA, R.; NOBLE, J.; MARSHALL, S. Developing a grounded theory to explain the practices of self-organizing agile teams. Empirical Software Engineering, p.609-639, 2012.

MOORMAN, C.; MINER, A. S. The Convergence of Planning and Execution: Improvisation in New Product Development. Journal of Marketing, p. 1-20, 1998.

ROZENFELD, H.; FORCELLINI, F. A.; AMARAL, D. C.; TOLEDO, J. C.; DA SILVA, S. L.; ALLIPRANDINI, D. H.; SCALICE, R. K. Gestão de desenvolvimento de produtos: uma referência para a melhoria do processo. São Paulo: Saraiva, 2006.

STRAY, V.; SJØBERG, D. I. K.; DYBÅ, T. The daily stand-up meeting: A grounded theory study. The Journal of Systems and Software, v. 114, p.101-124, 2016.

TAROZZI, M. O que é a Grounded Theory? Metodologia de pesquisa e de teoria fundamentada nos dados. Petrópolis: Vozes, 2011.

ULRICH, K. T.; EPPINGER, S. D. Product design and development. New York: McGrawHill, 2012. 